\title{
Solving Singular Boundary Value Problems by Optimal Homotopy Asymptotic Method
}

\author{
S. Zuhra, ${ }^{1}$ S. Islam, ${ }^{2}$ M. Idrees, ${ }^{2}$ Rashid Nawaz, ${ }^{1,2}$ I. A. Shah, ${ }^{2}$ and H. Ullah ${ }^{1}$ \\ ${ }^{1}$ Department of Mathematics, Islamia College University Peshawar, Pakistan \\ ${ }^{2}$ Department of Mathematics, Abdul Wali Khan University, Mardan, Khyber Pakhtunkhwa, Pakistan
}

Correspondence should be addressed to H. Ullah; hakeemullah1@gmail.com

Received 14 February 2014; Revised 1 June 2014; Accepted 2 June 2014; Published 29 June 2014

Academic Editor: Domiri D. Ganji

Copyright (C) 2014 S. Zuhra et al. This is an open access article distributed under the Creative Commons Attribution License, which permits unrestricted use, distribution, and reproduction in any medium, provided the original work is properly cited.

\begin{abstract}
In this paper, optimal homotopy asymptotic method (OHAM) for the semianalytic solutions of nonlinear singular two-point boundary value problems has been applied to several problems. The solutions obtained by OHAM have been compared with the solutions of another method named as modified adomain decomposition (MADM). For testing the success of OHAM, both of the techniques have been analyzed against the exact solutions in all problems. It is proved by this paper that solutions of OHAM converge rapidly to the exact solution and show most effectiveness as compared to MADM.
\end{abstract}

\section{Introduction}

Nonlinear singular boundary values problem (SBVP) is of considerable importance in the modeling of many branches of mathematical physics and engineering i-e fluid mechanics, quantum mechanics, astrophysics and so forth. Nonlinear problems are difficult to solve as compared to linear problems but the singularity of such nonlinear problem becomes more difficult to solve by researcher. Such type of problems have been successfully solved by modified adomian decomposition method $[1,2]$, but the convergence rate of its series goes gradually to accurate solution. Recently a new growing method has been introduced by Marinca et al. [3-6], for approximate solution of the problems of thin film flow of a fourth grade fluid on a cylinder surface and for understanding the behavior of nonlinear mechanical vibration of an electrical machines. OHAM attracted great attention of many researchers to solve a large class of linear and nonlinear differential equations and so forth; Idrees et al. extended OHAM by applying on initial and boundary values problems of ODEs and PDEs [7-10]. Iqbal and Javed [11] applied OHAM for the analytical solution of singular Lane-Emden type equations. The goal of this paper is to apply the proposed method to compute best approximate solutions of nonlinear singular boundary values problems. This paper is arranged in four sections; the first section of the paper describes the introduction, the second section of the paper is about the basic idea of OHAM, and the third section presents numerical results to demonstrate the efficiency of OHAM, and the last section of the paper is the conclusion.

\section{Basic Mathematical Theory of Optimal Homotopy Asymptotic Method}

The framework of OHAM is given in few steps.

(i) Consider the undertaken differential equation

$$
\begin{gathered}
\mathscr{L}(w(\xi, \tau))+\mathscr{N}(w(\xi, \tau))+\mathscr{g}(\xi, \tau)=0, \\
\mathscr{B}\left(w, w_{\tau}\right)=0,
\end{gathered}
$$

where $\xi$ and $\tau$ are independent variables and $\mathscr{L}(w(\xi, \tau))$ and $\mathcal{N}(w(\xi, \tau))$ are linear and nonlinear part of (1), respectively. Simply linear operator is denoted by $\mathscr{L}$ and nonlinear by $\mathscr{N}$, $w(\xi, \tau)$ is taken as an unknown function, $\mathscr{g}(x, \tau)$ is considered as known function, and $\mathscr{B}\left(w, w_{\tau}\right)$ yield corresponding initial condition. Now 
(ii) According to OHAM, an optimal homotopy, $\varphi(\xi, q)$ : $\mathscr{B} \times[0,1] \rightarrow R$ can be built, fulfilling homotopy equation:

$$
\begin{gathered}
(1-d)[\mathscr{L}(\varphi(\xi, \tau ; d))+\mathscr{g}(\xi, \tau)] \\
=\mathscr{H}(d)[\mathscr{L}(\varphi(\xi, \tau ; d))+\mathscr{g}(\xi, \tau)+\mathscr{N}(\varphi(\xi, \tau ; d))], \\
\mathscr{B}(\varphi(\xi, \tau ; d))=0,
\end{gathered}
$$

$\mathscr{H}(d)$ is auxiliary function with $d \in[0,1]$, an embedding parameter, such that

$$
\mathscr{H}(d)= \begin{cases}\sum_{j=1}^{\infty} K_{j} d^{j}, & d \neq 0, \\ 0, & d=0 .\end{cases}
$$

$d$ varies from 0 to 1 and the solution $\varphi(\xi, \tau ; d)$ varies from $w_{0}(\xi, \tau)$ to the final solution $w(\xi, \tau)$, where $w_{0}(\xi, \tau)$ is evaluated from (2) for $d=0$ :

$$
\mathscr{L}\left(w_{0}(\xi, \tau)\right)+\mathscr{Q}(\xi, \tau)=0, \quad \mathscr{B}\left(w_{0}, w_{\xi}\right)=0 .
$$

$\mathscr{H}(d)$ is reserved in the series as below:

$$
\mathscr{H}(d)=d K_{1}+d^{2} K_{2}+d^{3} K_{3}+\cdots
$$

(iii) Next $\varphi\left(\xi, \tau ; d: K_{i}\right)$ can be spread out by Taylor's series about the parameter $d$ :

$$
\begin{array}{r}
\varphi\left(\xi, \tau ; d: K_{i}\right)=w_{0}(\xi, \tau)+\sum_{k \geq 1} w_{k}\left(\xi, \tau ; K_{i}\right) d^{k} \\
i=1,2, \ldots
\end{array}
$$

$K_{1}, K_{2}, \ldots$, cause the accurateness and convergence of (6) and if it converges at $d=1$ then we have the following (see [5]):

$$
\varphi\left(\xi, \tau ; K_{i}\right)=w_{0}(\xi, \tau)+\sum_{k=1}^{m} w_{k}\left(\xi, \tau ; K_{i}\right) d^{k}, \quad i=1,2, \ldots, m
$$

Insert (6) into homotopy formula (2) and comparing like powers of $d$, the original nonlinear problem transferred into a sequence of linear problems, we obtained zeroth order (4), first order (8), second order (9), and $K$ th order (10) as below:

$$
\mathscr{L}\left(w_{1}(\xi, \tau)\right)=K_{1} \mathcal{N}_{0}\left(w_{0}(\xi, \tau)\right), \quad \mathscr{B}\left(w_{1}, \frac{d w_{1}}{d \xi}\right)=0,
$$

$$
\left\{\begin{array}{l}
\mathscr{L}\left(w_{2}(\xi, \tau)\right)-\mathscr{L}\left(w_{1}(\xi, \tau)\right) \\
=K_{2} \mathscr{N}_{0}\left(w_{0}(\xi, \tau)\right) \\
\quad+K_{1}\left(\mathscr{L}\left(w_{1}(\xi, \tau)\right)+\mathscr{N}_{1}\left(w_{0}(\xi, \tau)\right), w_{1}(\xi, \tau)\right), \\
\mathscr{B}\left(w_{2}, \frac{d w_{2}}{d \xi}\right)=0
\end{array}\right\}
$$

$$
\left\{\begin{array}{c}
\mathscr{L}\left(w_{k}(\xi, \tau)\right)-\mathscr{L}\left(w_{k-1}(\xi, \tau)\right) \\
=K_{k} \mathscr{N}_{0}\left(w_{0}(\xi, \tau)\right) \\
+\sum_{i=1}^{k-1} K_{i}\left(\mathscr{L}\left(w_{k-1}(\xi, \tau)\right)\right. \\
+\mathscr{N}_{k-1}\left(w_{0}(\xi, \tau),\right. \\
\left.\left.w_{1}(\xi, \tau), \ldots, w_{k-i}(\xi, \tau)\right)\right), \\
\mathscr{B}\left(w_{k}, \frac{d w_{k}}{d \xi}\right)=0 \quad k=2,3,4, \ldots
\end{array}\right\}
$$

(iv) The resultant linear problems can now be solved and their solutions are used to construct $K$ th order solution that comprises $K_{i}$ of the original problem through (7). Then by substituting (7) into (1), we get the following residual:

$$
\begin{aligned}
R\left(\xi, \tau ; K_{i}\right)= & \mathscr{L}\left(\widetilde{w}^{(m)}\left(\xi, \tau ; K_{i}\right)\right)+\mathscr{g}(\xi, \tau) \\
& +\mathscr{N}\left(\widetilde{w}^{(m)}\left(\xi, \tau ; K_{i}\right)\right)
\end{aligned}
$$

when $R\left(\xi, \tau ; K_{i}\right)=0$, for some values of $K_{i}$ then $\mathscr{L}\left(\widetilde{w}^{(m)}(\xi\right.$, $\left.\tau ; K_{i}\right)$ ) will agree with the exact solution. Though, this does not occur generally, especially, in nonlinear problems. Therefore, optimal values of the auxiliary constants $K_{1}, K_{2}, \ldots, K_{n}$ are calculated for minimizing the following functional $J$ (see [5]):

$$
J\left(K_{1}, K_{2}, \ldots, K_{n}\right)=\int_{0}^{1} R^{2}\left(\xi, K_{1}, K_{2}, \ldots, K_{m}\right) d \xi
$$

Therefore, the unknown constants $K_{i}(i=1,2, \ldots m)$ can be optimally identified from the following conditions (see [5]):

$$
\frac{\partial J}{\partial K_{1}}=\frac{\partial J}{\partial K_{2}}=\cdots=\frac{\partial J}{\partial K_{m}}=0
$$

With these known values of the auxiliary constants the approximate solution (7) is now well determined. 


\section{Application of OHAM}

OHAM is implemented on three models of singular boundary value problems in order to show the accuracy of this method.

Model 3.1. Consider second order singular boundary value problem [1]:

$$
w^{\prime \prime}(\xi)-\frac{1}{\xi} w^{\prime}(\xi)=\frac{\xi^{2}}{3} w^{5}(\xi),
$$

subject to boundary conditions

$$
w(0)=1, \quad w^{\prime}(1)=-0.216506
$$

with exact solution

$$
w(\xi)=\left(1+\frac{\xi^{2}}{3}\right)^{-1 / 2} .
$$

Zeroth Order Problem. Consider the following:

$$
\begin{gathered}
w_{0}^{\prime \prime}(x)=\frac{w_{0}^{\prime}(\xi)}{\xi}, \\
w_{0}(0)=1, \quad w_{0}^{\prime}(1)=-0.216506 .
\end{gathered}
$$

Its solution is

$$
w_{0}(\xi)=1-0.108253 \xi^{2} .
$$

First Order Problem. Consider the following:

$$
\begin{gathered}
w_{1}^{\prime \prime}(\xi)-w_{0}^{\prime \prime}(\xi)\left(1+K_{1}\right)-\frac{w_{1}^{\prime}(\xi)}{\xi}+\frac{w_{0}^{\prime}(\xi)}{\xi}\left(1+K_{1}\right) \\
+0.3333333 \xi^{2} K_{1} w_{0}^{5}(\xi)=0 \\
w_{1}(0)=0, \quad w_{1}^{\prime}(1)=0 .
\end{gathered}
$$

Its solution is

$$
\begin{aligned}
w_{1}\left(\xi, K_{1}\right) & \\
=( & 0.0637828 \xi^{2}-0.0416667 \xi^{4}+0.00751757 \xi^{6} \\
& -0.000813799 \xi^{8}+0.0000528577 \xi^{10} \\
& \left.-1.90734 \times 10^{-6} \xi^{12}+2.94964 \times 10^{-8} \xi^{14}\right) K_{1} .
\end{aligned}
$$

Second Order Problem. Consider the following:

$$
\begin{aligned}
& w_{2}^{\prime \prime}(\xi)-w_{1}^{\prime \prime}(\xi)\left(1+K_{1}\right)-K_{2} w_{0}^{\prime \prime}(\xi) \\
& -\frac{w_{2}^{\prime}(\xi)}{\xi}+\frac{w_{1}^{\prime}(\xi)}{\xi}\left(1+K_{1}\right)+\frac{K_{2} w_{0}^{\prime}(\xi)}{\xi} \\
& +0.3333333 \xi^{2} K_{2} w_{0}^{5}(\xi) \\
& +1.6666667 \xi^{2} K_{1} w_{0}^{4}(\xi) w_{1}(\xi)=0 \\
& w_{2}(0)=0, \quad w_{2}^{\prime}(1)=0 .
\end{aligned}
$$

Its solution is

$$
\begin{aligned}
& w_{1}\left(\xi, K_{1}, K_{2}\right) \\
&=K_{1}\left(0.0637828 \xi^{2}-0.0416667 \xi^{4}+0.00751757 \xi^{6}\right. \\
&-0.000813799 \xi^{8}+0.0000528577 \xi^{10} \\
&\left.-1.90734 \times 10^{-6} \xi^{12}+2.94964 \times 10^{-8} \xi^{14}\right) \\
&+K_{1}^{2}\left(0.0700404 \xi^{2}-0.0416667 \xi^{4}+0.00308821 \xi^{6}\right. \\
&+0.00159194 \xi^{8}-0.000573068 \xi^{10} \\
&+0.0000997917 \xi^{12}-0.000011419 \xi^{14} \\
&+9.36638 \times 10^{-7} \xi^{16} \\
&-5.633 \times 10^{-8} \xi^{18}+2.43915 \times 10^{-9} \xi^{20} \\
&-7.20125 \times 10^{-11} \xi^{22} \\
&\left.+1.29926 \times 10^{-12} \xi^{24}-1.08191 \times 10^{-14} \xi^{26}\right) \\
&+K_{2}\left(0.0637828 \xi^{2}-0.0416667 \xi^{4}+0.00751757 \xi^{6}\right. \\
&-0.000813799 \xi^{8}+0.0000528577 \xi^{10} \\
&\left.-1.90734 \times 10^{-6} \xi^{12}+2.94964 \times 10^{-8} \xi^{14}\right)
\end{aligned}
$$

Solve (17) to (22) to obtain approximate solution in the form of

$$
\widetilde{w}\left(\xi, K_{1}, K_{2}\right)=w_{0}(\xi)+w_{1}\left(\xi, K_{1}\right)+w_{2}\left(\xi, K_{1}, K_{2}\right) .
$$

To determine constant values, $K_{1}$ and $K_{2}$, apply the method of least square (12), (13) where

$$
\begin{aligned}
& K_{1}=-0.902176342508146, \\
& K_{2}=-0.004742918346272 .
\end{aligned}
$$

By substituting values of $K_{1}$ and $K_{2}$ in (22), we achieved the approximate solution of OHAM.

Model 3.2. Consider the following third order nonlinear boundary value problem [2]:

$$
w^{\prime \prime \prime}(\xi)-\frac{2}{x} w^{\prime \prime}(\xi)-w(\xi)-w^{2}(\xi)=h(\xi),
$$

with boundary conditions

$$
w(0)=0, \quad w^{\prime}(0)=0, \quad w(1)=2.71828182,
$$

where

$$
h(\xi)=7 \xi^{2} e^{\xi}+6 \xi e^{\xi}-6 e^{\xi}-\xi^{6} e^{2 \xi} .
$$


Zeroth Order Problem. Consider the following:

$$
\begin{aligned}
w_{0}^{\prime \prime \prime}(\xi)= & \frac{2 w_{0}^{\prime \prime}(\xi)}{\xi}-6+10 \xi^{2}+9 \xi^{3}+\frac{17}{4} \xi^{4}+\frac{41}{30} \xi^{5}-\frac{2}{3} \xi^{6} \\
& -\frac{325}{168} \xi^{7}-\frac{5729}{2880} \xi^{8}-\frac{20137}{15120} \xi^{9}-\frac{67181}{100800} \xi^{10} \\
w_{0}(0)= & 0, \quad w_{0}^{\prime}(0)=0, \quad w_{0}(1)=2.71828182 .
\end{aligned}
$$

Its solution is

$$
\begin{aligned}
w_{0}(\xi)= & \xi^{3}+1.0382 \xi^{4}+0.5 \xi^{5}+0.15 \xi^{6}+0.0337302 \xi^{7} \\
& +0.00610119 \xi^{8}-0.00185185 \xi^{9}-0.00358245 \xi^{10} \\
& -0.00258342 \xi^{11}-0.00126119 \xi^{12}-0.0004747 \xi^{13}
\end{aligned}
$$

First Order Problem. Consider the following:

$$
\begin{gathered}
w_{1}^{\prime \prime \prime}(\xi)-w_{0}^{\prime \prime \prime}(\xi)\left(1+K_{1}\right)-\frac{2 w_{1}^{\prime \prime}(\xi)}{\xi}+\frac{2 w_{0}^{\prime \prime}(\xi)}{\xi}\left(1+K_{1}\right) \\
+w_{0}^{2}(\xi) K_{1}+w_{0}(\xi) K_{1} \\
+\left(-6+10 \xi^{2}+9 \xi^{3}+\frac{17}{4} \xi^{4}+\frac{41}{30} \xi^{5}-\frac{2}{3} \xi^{6}-\frac{325}{168} \xi^{7}\right. \\
\left.-\frac{5729}{2880} \xi^{8}-\frac{20137}{15120} \xi^{9}-\frac{67181}{100800} \xi^{10}\right)\left(1+K_{1}\right)=0, \\
w_{1}(0)=0, \quad w_{1}^{\prime}(0)=0, \quad w_{1}(1)=0 .
\end{gathered}
$$

Its solution is

$$
\begin{aligned}
w_{1}\left(\xi, K_{1}\right) & \\
= & 7.544 \times 10^{-18} \xi^{4}-7.04904 \times 10^{-18} \xi^{7} \\
& -9.91271 \times 10^{-19} \xi^{8}-3.08395 \times 10^{-19} \xi^{9} \\
+ & 4.11194 \times 10^{-19} \xi^{10}+2.8837 \times 10^{-19} \xi^{11} \\
+ & 1.05135 \times 10^{-19} \xi^{12} \\
+ & K_{1}\left(0.0388054 \xi^{4}-0.0166667 \xi^{6}-0.00823971 \xi^{7}\right. \\
& -0.00223214 \xi^{8}-0.00319444 \xi^{9} \\
& -0.00390766 \xi^{10}-0.00270645 \xi^{11} \\
& -0.00126549 \xi^{12}-0.000445398 \xi^{13} \\
& -0.000126185 \xi^{14}-0.000027677 \xi^{15} \\
& -1.64425 \times 10^{-6} \xi^{16} \\
+ & 3.24922 \times 10^{-6} \xi^{16}+2.71079 \times 10^{-6} \xi^{18} \\
+ & 1.42573 \times 10^{-6} \xi^{19}+5.40486 \times 10^{7} \xi^{20} \\
+ & 1.49526 \times 10^{-7} \xi^{21}+2.95509 \times 10^{-8} \xi^{22}
\end{aligned}
$$

$$
\begin{aligned}
& +2.60148 \times 10^{-9} \xi^{23}-1.57505 \times 10^{-9} \xi^{24} \\
& -1.38639 \times 10^{-9} \xi^{25}-6.93534 \times 10^{-10} \xi^{26} \\
& -2.50421 \times 10^{-10} \xi^{27}-6.59925 \times 10^{-11} \xi^{28} \\
& \left.-1.11005 \times 10^{-11} \xi^{29}\right) .
\end{aligned}
$$

Second Order Problem. Consider the following:

$$
\begin{aligned}
& \xi y_{2}^{\prime \prime \prime}(x)-\xi K_{1} y_{1}^{\prime \prime \prime}(\xi)-\xi y_{1}^{\prime \prime \prime}(\xi)-\xi C_{2} y_{0}^{\prime \prime \prime}(\xi)+2 K_{1} y_{1}^{\prime \prime}(\xi) \\
& +2 K_{2} y_{0}^{\prime \prime}(\xi)+2 \xi K_{1} y_{0}(\xi) y_{1}(\xi)+\xi K_{1} y_{1}(\xi) \\
& +\xi K_{2} y_{0}^{2}(\xi)+\xi K_{2} y_{0}(\xi)-2 y_{2}^{\prime \prime}(\xi)+2 y_{1}^{\prime \prime}(\xi) \\
& -6 \xi K_{2}+10 \xi^{3} K_{2}+9 \xi^{4} K_{2}+\frac{17}{4} \xi^{5} K_{2}+\frac{41}{30} \xi^{6} K_{2} \\
& -\frac{2}{3} \xi^{7} K_{2}-\frac{325}{168} \xi^{8} K_{2}-\frac{5729}{2880} \xi^{9} K_{2} \\
& -\frac{20137}{15120} \xi^{10} K_{2}-\frac{67181}{100800} \xi^{11} K_{2}=0, \\
& w_{2}(0)=0, \quad w_{2}^{\prime}(0)=0, \quad w_{2}(1)=0 .
\end{aligned}
$$

Its solution is

$$
\begin{aligned}
& w_{2}\left(\xi, K_{1}, K_{2}\right) \\
& =7.544 \times 10^{-18} \xi^{4}-7.04904 \times 10^{-18} \xi^{7} \\
& -9.91271 \times 10^{-19} \xi^{8}-3.08395 \times 10^{-19} \xi^{9} \\
& +4.11194 \times 10^{-19} \xi^{10}+2.8837 \times 10^{-19} \xi^{11} \\
& +1.05135 \times 10^{-19} \xi^{12}+1.05135 \times 10^{-19} \xi^{13} \\
& +K_{1}\left(0.0388054 \xi^{4}-0.0166667 \xi^{6}\right. \\
& -0.00823971 \xi^{7}-0.00223214 \xi^{8}-0.00319444 \xi^{9} \\
& -0.00390766 \xi^{10}-0.00270645 \xi^{11} \\
& -0.00126549 \xi^{12}-0.000445398 \xi^{13} \\
& -0.000126185 \xi^{14}-0.000027677 \xi^{15} \\
& -1.64425 \times 10^{-6} \xi^{16}+3.24922 \times 10^{-6} \xi^{16} \\
& +2.71079 \times 10^{-6} \xi^{18}+1.42573 \times 10^{-6} \xi^{19} \\
& +5.40486 \times 10^{7} \xi^{20}+1.49526 \times 10^{-7} \xi^{21} \\
& +2.95509 \times 10^{-8} \xi^{22}+2.60148 \times 10^{-9} \xi^{23} \\
& -1.57505 \times 10^{-9} \xi^{24}-1.38639 \times 10^{-9} \xi^{25} \\
& -6.93534 \times 10^{-10} \xi^{26}-2.50421 \times 10^{-10} \xi^{27} \\
& \left.-6.59925 \times 10^{-11} \xi^{28}-1.11005 \times 10^{-11} \xi^{29}\right)
\end{aligned}
$$




$$
\begin{aligned}
& +K_{1}^{2}\left(0.03692188 \xi^{4}-0.0166667 \xi^{6}\right. \\
& -0.00854769 \xi^{7}-0.00223214 \xi^{8} \\
& -0.0031481510 \xi^{9}-0.00403613 \xi^{10} \\
& -0.0028082 \xi^{11}-0.00126764 \xi^{12} \\
& -0.00041452 \xi^{13}-0.000105125 \xi^{14} \times 10^{-6} \xi^{16} \\
& +8.49649 \times 10^{-6} \xi^{17}+5.8467 \times 10^{-6} \xi^{18} \\
& +2.90517 \times 10^{-6} \xi^{19}+1.10585 \times 10^{-6} \xi^{20} \\
& +3.2565 \times 10^{-7} \xi^{21}+7.18826 \times 10^{-8} \xi^{22} \\
& +6.70135 \times 10^{-9} \xi^{23}-5.45808 \times 10^{-9} \xi^{24} \\
& -4.99343 \times 10^{-9} \xi^{25}-2.6526 \times 10^{-9} \xi^{26} \\
& -1.05107 \times 10^{-9} \xi^{27}-3.27247 \times 10^{-10} \xi^{28} \\
& +7.96076 \times 10^{-11} \xi^{29}-1.3044 \times 10^{-11} \xi^{30} \\
& -4.36977 \times 10^{-13} \xi^{31}-1.22809 \times 10^{-12} \xi^{32} \\
& +8.15668 \times 10^{-13} \xi^{33}+3.55887 \times 10^{-13} \xi^{34} \\
& +1.20393 \times 10^{-13} \xi^{35}+3.18368 \times 10^{-14} \xi^{36} \\
& +6.13738 \times 10^{-15} \xi^{37}+5.19104 \times 10^{-16} \xi^{38} \\
& -2.50795 \times 10^{-16} \xi^{39}-1.86591 \times 10^{-16} \xi^{40} \\
& -8.03133 \times 10^{-17} \xi^{41}-2.61417 \times 10^{-17} \xi^{42} \\
& -6.5531 \times 10^{-18} \xi^{43}-1.19784 \times 10^{-18} \xi^{44} \\
& -1.2982 \times 10^{-19} \xi^{45}-0.0000168305 \xi^{15} \\
& +5.60083 \times 10^{-6} \xi^{16}+8.49649 \times 10^{-6} \xi^{17} \\
& +5.8467 \times 10^{-6} \xi^{18}+2.90517 \times 10^{-6} \xi^{19} \\
& +1.10585 \times 10^{-6} \xi^{20}+3.2565 \times 10^{-7} \xi^{21} \\
& +7.18826 \times 10^{-8} \xi^{22}+6.70135 \times 10^{-9} \xi^{23} \\
& -5.45808 \times 10^{-9} \xi^{24}-4.99343 \times 10^{-9} \xi^{25} \\
& -2.6526 \times 10^{-9} \xi^{26}-1.05107 \times 10^{-9} \xi^{27} \\
& -3.27247 \times 10^{-10} \xi^{28}+7.96076 \times 10^{-11} \xi^{29} \\
& -1.3044 \times 10^{-11} \xi^{30}-4.36977 \times 10^{-13} \xi^{31} \\
& -1.22809 \times 10^{-12} \xi^{32}+8.15668 \times 10^{-13} \xi^{33} \\
& +3.55887 \times 10^{-13} \xi^{34}+1.20393 \times 10^{-13} \xi^{35} \\
& +3.18368 \times 10^{-14} \xi^{36}+6.13738 \times 10^{-15} \xi^{37} \\
& +5.19104 \times 10^{-16} \xi^{38}-2.50795 \times 10^{-16} \xi^{39}
\end{aligned}
$$

$$
\begin{aligned}
- & 1.86591 \times 10^{-16} \xi^{40}-8.03133 \times 10^{-17} \xi^{41} \\
- & 2.61417 \times 10^{-17} \xi^{42}-6.5531 \times 10^{-18} \xi^{43} \\
- & \left.1.19784 \times 10^{-18} \xi^{44}-1.2982 \times 10^{-19} \xi^{45}\right) \\
+K_{2}( & 0.0388054 \xi^{4}-0.0166667 \xi^{6} \\
& -0.00823971 \xi^{7}-0.00223214 \xi^{8} \\
& -0.00319444 \xi^{9}-0.00390766 \xi^{10} \\
& -0.00270645 \xi^{11}-0.00126549 \xi^{12} \\
& -0.000445398 \xi^{13}-0.000126185 \xi^{14} \\
& -0.000027677 \xi^{15}-1.64425 \times 10^{-6} \xi^{16} \\
+ & 3.24922 \times 10^{-6} \xi^{16}+2.71079 \times 10^{-6} \xi^{18} \\
+ & 1.42573 \times 10^{-6} \xi^{19}+5.40486 \times 10^{7} \xi^{20} \\
& +1.49526 \times 10^{-7} \xi^{21}+2.95509 \times 10^{-8} \xi^{22} \\
+ & 2.60148 \times 10^{-9} \xi^{23}-1.57505 \times 10^{-9} \xi^{24} \\
& -1.38639 \times 10^{-9} \xi^{25}-6.93534 \times 10^{-10} \xi^{26} \\
& -2.50421 \times 10^{-10} \xi^{27}-6.59925 \times 10^{-11} \xi^{28} \\
& \left.-1.11005 \times 10^{-11} \xi^{29}\right) .
\end{aligned}
$$

Solving problem (28) to (33), in the form of

$$
\widetilde{w}\left(\xi, K_{1}, K_{2}\right)=w_{0}(\xi)+w_{1}\left(\xi, K_{1}\right)+w_{2}\left(\xi, K_{1}, K_{2}\right) .
$$

We obtained the constant values of $K_{1}$ and $K_{2}$ as

$$
\begin{gathered}
K_{1}=-0.615104986973096, \\
K_{2}=-0.11776184847135589 .
\end{gathered}
$$

Taylor series of the exact solution with order 10, which is given below

$$
\begin{aligned}
w(\xi)= & \xi^{3}+\xi^{4}+0.5 \xi^{5}+0.166666 \xi^{6}+0.041666666 \xi^{7} \\
& +0.008333 \xi^{8}+0.0013888 \xi^{9}+0.000198 \xi^{10}+\cdots
\end{aligned}
$$

Model 3.3. Consider the following third order singular boundary value problem [1]:

$$
w^{\prime \prime \prime}(\xi)-\frac{2}{\xi} w^{\prime \prime}(\xi)-w^{3}(\xi)=h(\xi),
$$

subject to boundary conditions

$$
w(0)=0, \quad w^{\prime}(0)=0, \quad w^{\prime}(1)=10.8371,
$$


where

$$
h(\xi)=7 \xi^{2} e^{\xi}+6 \xi e^{\xi}-6 e^{\xi}-x^{9} e^{3 \xi}+x^{3} e^{\xi} .
$$

Exact solution of (41) is

$$
w(\xi)=\xi^{3} e^{\xi} .
$$

Zeroth Order Problem. Consider the following:

$$
\begin{aligned}
& w_{0}^{\prime \prime \prime}(\xi)-\frac{2 w_{0}^{\prime \prime}(\xi)}{\xi}+6-10 \xi^{2}-10 \xi^{3}-5.25 \xi^{4} \\
& -1.86666666667 \xi^{5}-0.5 \xi^{6}-0.107142857143 \xi^{7} \\
& -0.0190972222224 \xi^{8}+0.9970899470899471 \xi^{9} \\
& +2.9996130952380953 \xi^{10}=0 \\
& w_{0}(0)=0, \quad w_{0}^{\prime}(0)=0, \quad w_{0}^{\prime}(1)=10.8731 .
\end{aligned}
$$

Its solution is

$$
\begin{aligned}
w_{0}(\xi)= & \xi^{3}+1.0097 \xi^{4}+0.5 \xi^{5}+0.166667 \xi^{6} \\
& +0.0416667 \xi^{7}+0.00833333 \xi^{8}+0.00138889 \xi^{9} \\
& +0.000198413 \xi^{10}+0.0000248016 \xi^{11} \\
& -0.000944214 \xi^{12}-0.00213648 \xi^{13}
\end{aligned}
$$

First Order Problem. Consider the following:

$$
\begin{aligned}
& w_{1}^{\prime \prime \prime}(\xi)-w_{0}^{\prime \prime \prime}(\xi)\left(1+K_{1}\right)-\frac{2 w_{1}^{\prime \prime}(\xi)}{\xi} \\
& +\frac{2 w_{0}^{\prime \prime}(\xi)}{\xi}\left(1+K_{1}\right)+w_{0}^{2}(\xi) K_{1} \\
& -\left(6-10 \xi^{2}-9 \xi^{3}-5.25 \xi^{4}\right. \\
& \quad-1.86666666667 \xi^{5}-0.5 \xi^{6}-0.107142857143 \xi^{7} \\
& \quad-0.0190972222224 \xi^{8}+0.9970899470899471 \xi^{9} \\
& \left.+2.9996130952380953 \xi^{10}\right)\left(1+K_{1}\right)=0, \\
& w_{1}(0)=0, \quad w_{1}^{\prime}(0)=0, \quad w_{1}^{\prime}(1)=0 .
\end{aligned}
$$

Its solution is

$$
\begin{aligned}
w_{1}\left(\xi, K_{1}\right) & \\
= & 2.96059 \times 10^{-16} \xi^{3}-2.07889 \times 10^{-16} \xi^{4} \\
& -7.04904 \times 10^{-18} \xi^{7}-1.98254 \times 10^{-18} \xi^{8} \\
& +2.56996 \times 10^{-20} \xi^{10}+9.01155 \times 10^{-21} \xi^{11} \\
& +6.32606 \times 10^{-19} \xi^{13}
\end{aligned}
$$

$$
+K_{1}\left(2.96059 \times 10^{-16} \xi^{3}+0.0348465 \xi^{4}\right.
$$$$
-7.04904 \times 10^{-18} \xi^{7}-1.98254 \times 10^{-18} \xi^{8}
$$$$
+2.56996 \times 10^{-20} \xi^{10}+9.01155 \times 10^{-21} \xi^{11}
$$$$
-0.00094697 \xi^{12}-0.00215765 \xi^{13}
$$$$
-0.000250492 \xi^{14}-0.00197358 \xi^{15}
$$$$
-0.00118551 \xi^{16}-0.000578225 \xi^{17}
$$$$
-0.000238163 \xi^{18}-0.0000850952 \xi^{19}
$$$$
-0.0000268863 \xi^{20}-7.225 \times 10^{-6} \xi^{21}
$$$$
-5.00972 \times 10^{-7} \xi^{22}
$$$$
+1.4809 \times 10^{-6} \xi^{23}+1.41769 \times 10^{-6} \xi^{24}
$$$$
+8.1403 \times 10^{-7} \xi^{25}+3.49615 \times 10^{-7} \xi^{26}
$$$$
+1.21188 \times 10^{-7} \xi^{27}+3.53307 \times 10^{-8} \xi^{28}
$$$$
+8.89992 \times 10^{-9} \xi^{29}+1.85617 \times 10^{-9} \xi^{30}
$$$$
-1.99788 \times 10^{-10} \xi^{31}-9.12587 \times 10^{-10} \xi^{32}
$$$$
-6.52991 \times 10^{-10} \xi^{33}-2.65181 \times 10^{-10} \xi^{34}
$$$$
-7.59661 \times 10^{-11} \xi^{35}-1.67268 \times 10^{-11} \xi^{36}
$$$$
-2.98912 \times 10^{-12} \xi^{37}-4.49394 \times 10^{-13} \xi^{38}
$$$$
-4.19389 \times 10^{-14} \xi^{39}+9.57023 \times 10^{-14} \xi^{40}
$$$$
\left.+2.1308 \times 10^{-13} \xi^{41}+1.49031 \times 10^{-13} \xi^{42}\right) .
$$

Second Order Problem. Consider the following:

$$
\begin{aligned}
& w_{2}^{\prime \prime \prime}(\xi)-w_{1}^{\prime \prime \prime}(\xi)\left(1+K_{1}\right)-w_{0}^{\prime \prime \prime}(\xi) K_{2} \\
& -\frac{2 w_{2}^{\prime \prime}(\xi)}{\xi}+\frac{2 w_{1}^{\prime \prime}(\xi)}{\xi}\left(1+K_{1}\right)+\frac{2 K_{2} w_{0}^{\prime \prime}(\xi)}{\xi} \\
& +3 K_{1} w_{0}^{2}(\xi) w_{1}(\xi)+K_{2} w_{0}^{3}(\xi)-w_{1}^{\prime \prime \prime}(\xi) \\
& -w_{0}^{\prime \prime \prime}(\xi)\left(1+K_{1}\right) \\
& -\left(6-10 \xi^{2}-10 \xi^{3}-5.25 \xi^{4}-1.86666666667 \xi^{5}\right. \\
& \quad-0.5 \xi^{6}-0.107142857143 \xi^{7}-0.0190972222224 \xi^{8} \\
& +0.9970899470899471 \xi^{9} \\
& \left.+2.9996130952380953 \xi^{10}\right) K_{2}=0, \\
& w_{2}(0)=0, \quad w_{2}^{\prime}(0)=0, \quad w_{2}^{\prime}(1)=0 .
\end{aligned}
$$


TABLE 1: Comparison of second order OHAM solution with exact solution and third order MADM solution up to order 14.

\begin{tabular}{lccccc}
\hline$\xi$ & EXACT solution & MADM solution & OHAM solution & Absolute error MADM & Absolute error OHAM \\
\hline 0.1 & 0.998337 & 0.998336 & 0.998338 & $1.78094 \times 10^{-6}$ & $3.03759 \times 10^{-7}$ \\
0.2 & 0.993399 & 0.993392 & 0.9934 & $7.12004 \times 10^{-6}$ & $1.00144 \times 10^{-6}$ \\
0.3 & 0.985329 & 0.985313 & 0.985331 & $1.59824 \times 10^{-5}$ & $1.59946 \times 10^{-7}$ \\
0.4 & 0.974355 & 0.974326 & 0.974356 & $2.82262 \times 10^{-5}$ & $1.66785 \times 10^{-6}$ \\
0.5 & 0.960769 & 0.960725 & 0.96077 & $4.34674 \times 10^{-5}$ & $1.14773 \times 10^{-6}$ \\
0.6 & 0.944911 & 0.94485 & 0.944911 & $6.08991 \times 10^{-5}$ & $3.98556 \times 10^{-7}$ \\
0.7 & 0.927145 & 0.927066 & 0.927145 & $7.91571 \times 10^{-5}$ & $1.05074 \times 10^{-7}$ \\
0.8 & 0.907842 & 0.907745 & 0.907841 & $9.65148 \times 10^{-5}$ & $3.56447 \times 10^{-7}$ \\
0.9 & 0.887358 & 0.887246 & 0.887357 & $1.12075 \times 10^{-4}$ & $1.45927 \times 10^{-6}$ \\
1 & 0.866031 & 0.865902 & 0.866026 & $1.29346 \times 10^{-4}$ & $5.62911 \times 10^{-6}$ \\
\hline
\end{tabular}

Second order OHAM solution gives encouraging results after comparing it with third order MADM solution.

TABLE 2: Comparison of OHAM solution with exact solution and MADM solution of order 10 .

\begin{tabular}{lccccc}
\hline$\xi$ & EXACT solution & MADM solution & OHAM solution & Absolute error MADM & Absolute error OHAM \\
\hline 0.1 & 0.00110519 & 0.00110515 & 0.00110517 & $1.87999 \times 10^{-8}$ & $2.64081 \times 10^{-14}$ \\
0.2 & 0.00977122 & 0.00977092 & 0.00977122 & $3.00822 \times 10^{-7}$ & $1.58487 \times 10^{-9}$ \\
0.3 & 0.0364462 & 0.036447 & 0.0364462 & $1.52339 \times 10^{-6}$ & $2.99917 \times 10^{-8}$ \\
0.4 & 0.0954768 & 0.095472 & 0.0954765 & $4.81805 \times 10^{-6}$ & $2.31584 \times 10^{-7}$ \\
0.5 & 0.20609 & 0.206078 & 0.206089 & $1.17777 \times 10^{-5}$ & $1.12844 \times 10^{-6}$ \\
0.6 & 0.393578 & 0.393553 & 0.393573 & $2.44717 \times 10^{-5}$ & $4.14866 \times 10^{-6}$ \\
0.7 & 0.690717 & 0.690671 & 0.690704 & $4.54747 \times 10^{-5}$ & $1.26327 \times 10^{-5}$ \\
0.8 & 1.13947 & 1.1394 & 1.13944 & $7.79179 \times 10^{-5}$ & $3.36654 \times 10^{-5}$ \\
0.9 & 1.79304 & 1.79292 & 1.79296 & $1.25578 \times 10^{-4}$ & $8.13233 \times 10^{-5}$ \\
1 & 2.71825 & 2.71806 & 2.71807 & $1.93023 \times 10^{-4}$ & $1.82278 \times 10^{-4}$ \\
\hline
\end{tabular}

Its solution is

$$
\begin{aligned}
& w_{2}\left(\xi, K_{1}, K_{2}\right) \\
&=2.96059 \times 10^{-16} \xi^{3}-2.07889 \times 10^{-16} \xi^{4} \\
&- 7.04904 \times 10^{-18} \xi^{7}-1.98254 \times 10^{-18} \xi^{8} \\
&+ 2.56996 \times 10^{-20} \xi^{10}+9.01155 \times 10^{-21} \xi^{11} \\
&+ 6.32606 \times 10^{-19} \xi^{13} \\
&+\left(5.92119 \times 10^{-16} \xi^{3}+0.0348465 \xi^{4}\right. \\
&-1.40981 \times 10^{-18} \xi^{7}-3.96508 \times 10^{-18} \xi^{8} \\
&+5.13992 \times 10^{-20} \xi^{10}+1.80231 \times 10^{-20} \xi^{11} \\
&-0.00094697 \xi^{12}-0.00215765 \xi^{13}-0.00250492 \xi^{14} \\
&-0.00197358 \xi^{15}-0.00118551 \xi^{16} \\
&-0.000578225 \xi^{17}-0.000238173 \xi^{18} \\
&-0.0000850952 \xi^{19}-0.0000268863 \xi^{20} \\
&-7.225 \times 10^{-6} \xi^{21}-5.00972 \times 10^{-7} \xi^{22} \\
& 1.4809 \times 10^{-6} \xi^{23}+1.41769 \times 10^{-6} \xi^{24}
\end{aligned}
$$

$$
\begin{aligned}
& +8.1403 \times 10^{-7} \xi^{25}+3.49615 \times 10^{-7} \xi^{26} \\
+ & 1.21188 \times 10^{-7} \xi^{27}+3.53307 \times 10^{-8} \xi^{28} \\
+ & 8.89992 \times 10^{-9} \xi^{29}+1.85617 \times 10^{-9} \xi^{30} \\
& -1.99788 \times 10^{-10} \xi^{31}-9.12587 \times 10^{-10} \xi^{32} \\
& -6.52991 \times 10^{-10} \xi^{33}-2.65181 \times 10^{-10} \xi^{34} \\
& -7.59661 \times 10^{-11} \xi^{35}-1.67268 \times 10^{-11} \xi^{36} \\
& -2.98912 \times 10^{-12} \xi^{37}-4.49394 \times 10^{-13} \xi^{38} \\
& -4.19389 \times 10^{-14} \xi^{39}+9.57023 \times 10^{-14} \xi^{40} \\
& \left.+2.1308 \times 10^{-13} \xi^{41}+1.49031 \times 10^{-13} \xi^{42}\right) K_{1} \\
+ & 2.96059 \times 10^{-16} \xi^{3}-0.0360497 \xi^{4} \\
& -7.04904 \times 10^{-18} \xi^{7} \\
& -1.98254 \times 10^{-18} \xi^{8}+2.56996 \times 10^{-20} \xi^{10} \\
+ & 9.01155 \times 10^{-21} \xi^{11}-0.00094697 \xi^{12} \\
& -0.0022321 \xi^{13}-0.00262092 \xi^{14} \\
& -0.00206498 \xi^{15}+0.00123426 \xi^{16}
\end{aligned}
$$


TABLE 3: Comparison of the series of OHAM solution with exact solution and MADM solution with order 10.

\begin{tabular}{lccccc}
\hline$\xi$ & EXACT solution & MADM solution & OHAM solution & Absolute error MADM & Absolute error OHAM \\
\hline 0.1 & 0.00110517 & 0.00110273 & 0.00110615 & $2.4423 \times 10^{-6}$ & $9.77862 \times 10^{-7}$ \\
0.2 & 0.00977122 & 0.00973215 & 0.00978687 & $3.90768 \times 10^{-5}$ & $1.56458 \times 10^{-5}$ \\
0.3 & 0.0364462 & 0.0362484 & 0.0365254 & $1.97827 \times 10^{-4}$ & $7.92069 \times 10^{-5}$ \\
0.4 & 0.0954768 & 0.0948515 & 0.0957271 & $6.25242 \times 10^{-4}$ & $2.50333 \times 10^{-4}$ \\
0.5 & 0.20609 & 0.204564 & 0.20670 & $1.52656 \times 10^{-3}$ & $6.11167 \times 10^{-4}$ \\
0.6 & 0.393578 & 0.390412 & 0.394845 & $3.16596 \times 10^{-3}$ & $1.26732 \times 10^{-3}$ \\
0.7 & 0.690717 & 0.684849 & 0.693064 & $5.86744 \times 10^{-3}$ & $2.34789 \times 10^{-3}$ \\
0.8 & 1.13947 & 1.12946 & 1.14348 & $1.00169 \times 10^{-2}$ & $4.00545 \times 10^{-3}$ \\
0.9 & 1.79304 & 1.77697 & 1.79946 & $1.6067 \times 10^{-2}$ & $6.41611 \times 10^{-3}$ \\
1 & 2.71825 & 2.69371 & 2.72805 & $2.45465 \times 10^{-2}$ & $9.77953 \times 10^{-3}$ \\
\hline
\end{tabular}

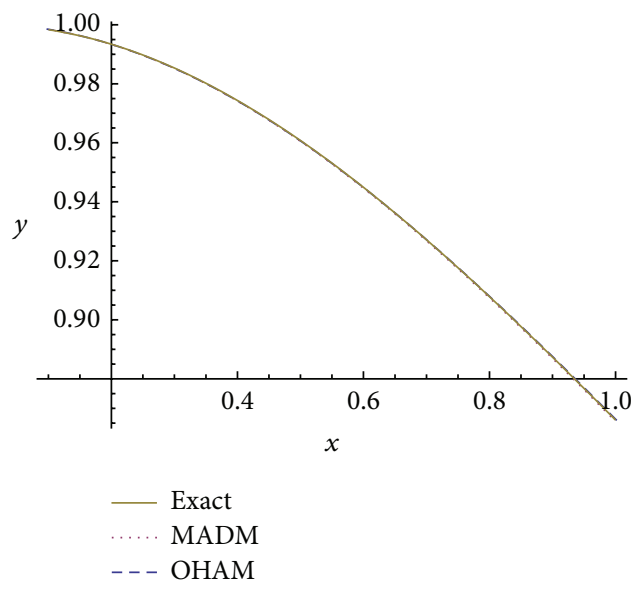

(a)

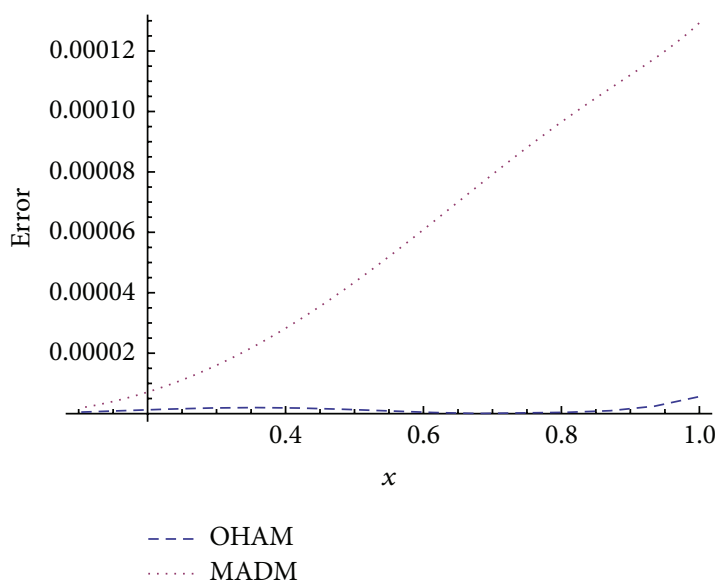

(b)

FIGURE 1: (a) Plot of exact, third order MADM, and second order OHAM solution of model 3.1. (b) Plot of absolute errors of OHAM and MADM with exact solutions.

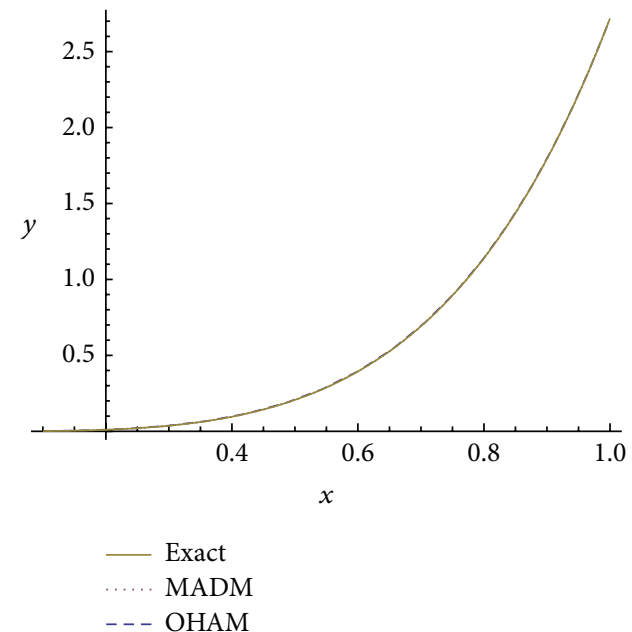

(a)

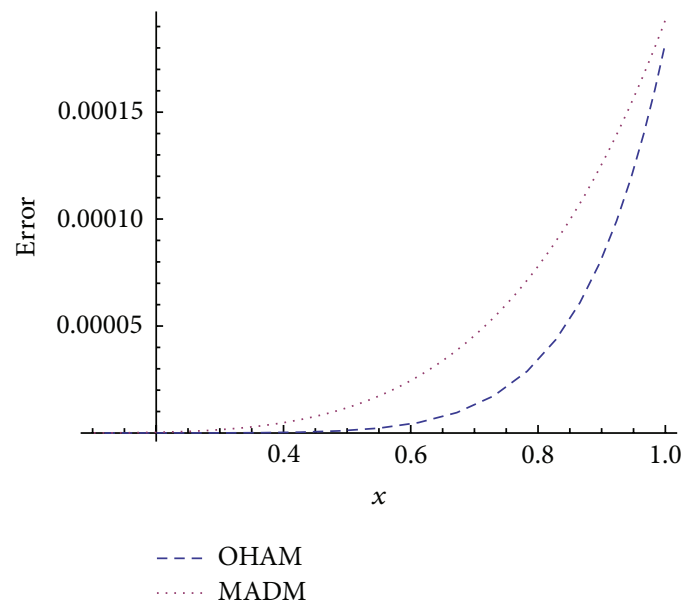

(b)

FIGURE 2: (a) Plot of Exact, second order MADM, second order OHAM solution of model 3.2. (b) Plot of Absolute errors of OHAM, MADM with exact solutions. 


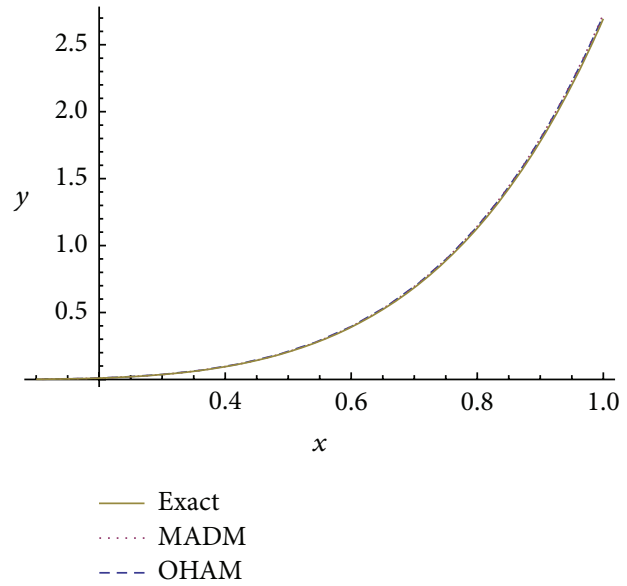

(a)

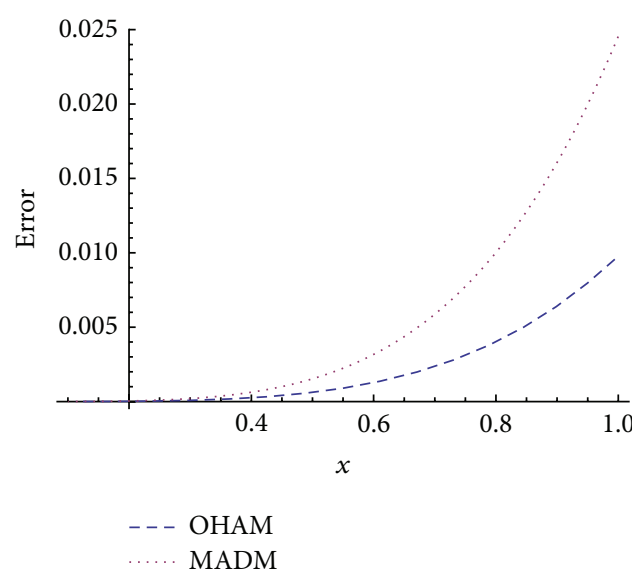

(b)

Figure 3: (a) Plot of Exact, third order MADM, second order OHAM solution of model 3.3. (b) Plot of Absolute errors of OHAM, MADM with exact solutions.

$$
\begin{aligned}
& -0.000598031 \xi^{17}-0.000244701 \xi^{18} \\
& -0.0000869099 \xi^{19} \\
& -0.0000273234 \xi^{20}-6.92013 \times 10^{-6} \xi^{21} \\
& +9.73386 \times 10^{-7} \xi^{22}+4.28328 \times 10^{-6} \xi^{23} \\
& +4.9078 \times 10^{-6} \xi^{24}+4.11114 \times 10^{-6} \xi^{25} \\
& +2.87739 \times 10^{-6} \xi^{26}+1.75577 \times 10^{-6} \xi^{27} \\
& +9.50658 \times 10^{-7} \xi^{28}+4.61508 \times 10^{-7} \xi^{29} \\
& +2.02183 \times 10^{-7} \xi^{30}+7.90011 \times 10^{-8} \xi^{31} \\
& +2.56676 \times 10^{-8} \xi^{32}+5.3291 \times 10^{-9} \xi^{33} \\
& -9.74128 \times 10^{-10} \xi^{34}-2.0341 \times 10^{-9} \xi^{35} \\
& -1.52924 \times 10^{-9} \xi^{36}-8.57227 \times 10^{-10} \xi^{37} \\
& -4.01512 \times 10^{-10} \xi^{38}-1.63899 \times 10^{-10} \xi^{39} \\
& -5.90489 \times 10^{-11} \xi^{40}-1.79114 \times 10^{-11} \xi^{41} \\
& -3.55181 \times 10^{-12} \xi^{42}+4.69351 \times 10^{-13} \xi^{43} \\
& +1.06979 \times 10^{-12} \xi^{44}+7.36983 \times 10^{-13} \xi^{45} \\
& +3.68208 \times 10^{-13} \xi^{46}+1.51155 \times 10^{-13} \xi^{47} \\
& +5.34052 \times 10^{-14} \xi^{48}+1.65282 \times 10^{-14} \xi^{49} \\
& +4.23773 \times 10^{-15} \xi^{50}+4.9859 \times 10^{-16} \xi^{51} \\
& -3.83336 \times 10^{-16} \xi^{52}-3.73432 \times 10^{-16} \xi^{53} \\
& -2.01066 \times 10^{-16} \xi^{54}-8.11761 \times 10^{-17} \xi^{55}
\end{aligned}
$$

$$
\begin{aligned}
& -2.66882 \times 10^{-17} \xi^{56}-7.4357 \times 10^{-18} \xi^{57} \\
& +1.78031 \times 10^{-18} \xi^{58}-3.07739 \times 10^{-19} \xi^{59} \\
& +6.87535 \times 10^{-20} \xi^{60}+1.26899 \times 10^{-19} \xi^{61} \\
& +7.41807 \times 10^{-20} \xi^{62}+2.6999 \times 10^{-20} \xi^{63} \\
& +7.17458 \times 10^{-21} \xi^{64}+1.49357 \times 10^{-21} \xi^{65} \\
& +2.55087 \times 10^{-22} \xi^{66}+3.52795 \times 10^{-23} \xi^{67} \\
& -2.35347 \times 10^{-24} \xi^{68}-1.39052 \times 10^{-23} \xi^{69} \\
& \left.-1.48117 \times 10^{-23} \xi^{70}-6.12864 \times 10^{-24} \xi^{71}\right) K_{1}^{2} .
\end{aligned}
$$

Solving problem (41) to (47), we obtained

$$
\widetilde{w}(\xi)=w_{0}(\xi)+w_{1}\left(\xi, K_{1}\right)+w_{2}\left(\xi, K_{1}, K_{2}\right) .
$$

We obtained the constant values of $K_{1}$ and $K_{2}$ as

$$
\begin{gathered}
K_{1}=2.7875178639324 \times 10^{-20}, \\
K_{2}=-7.889481663510912 \times 10^{-21} .
\end{gathered}
$$

Substituting constant values of $K_{1}$ and $K_{2}$ in (48) to obtain approximate solution of OHAM.

\section{Conclusion}

Optimal homotopy asymptotic method has been applied to obtain the approximate solution of singular boundary value problems. Results have been compared with modified adomian decomposition method and with the exact solutions of proposed models. Numerical results present in Tables 1, 2, and 3 showed that this technique is fast convergent and has 
remarkable low error than MADM. It is observed through represented plots in Figures 1(a), 2(a), and 3(a) that the presented technique is effective and reliable within bounded domain. Absolute errors plots by Figures 1(b), 2(b), and 3(b) showed the precise closeness of OHAM to exact results.

\section{Conflict of Interests}

The authors declare that there is no conflict of interests regarding the publication of this paper.

\section{References}

[1] Y. Q. Hasan and L. M. Zhu, "A note on the use of modified Adomian decomposition method for solving singular boundary value problems of higher-order ordinary differential equation," Communications in Nonlinear Science and Numerical Simulation, vol. 14, no. 8, pp. 3261-3265, 2009.

[2] Y. Q. Hasan and L. M. Zhu, "Solving singular boundary value problems of higher-order ordinary differential equations by modified Adomian decomposition method," Communications in Nonlinear Science and Numerical Simulation, vol. 14, no. 6, pp. 2592-2596, 2009.

[3] V. Marinca and N. Herişanu, "Application of optimal homotopy asymptotic method for solving nonlinear equations arising in heat transfer," International Communications in Heat and Mass Transfer, vol. 35, no. 6, pp. 710-715, 2008.

[4] V. Marinca, N. Herişanu, T. Dordea, and G. Madescu, "A new analytical approach to nonlinear vibration of an electrical machine," Proceedings of the Romanian Academy A: Mathematics Physics Technical Sciences Information Science, vol. 9, no. 3, pp. 229-236, 2008.

[5] V. Marinca, N. Herişanu, and I. Nemeş, "Optimal homotopy asymptotic method with application to thin film flow," Central European Journal of Physics, vol. 6, no. 3, pp. 648-653, 2008.

[6] V. Marinca, N. Herişanu, C. Bota, and B. Marinca, "An optimal homotopy asymptotic method applied to the steady flow of a fourth-grade fluid past a porous plate," Applied Mathematics Letters, vol. 22, no. 2, pp. 245-251, 2009.

[7] M. Idrees, S. Haq, and S. Islam, "Application of optimal homotopy asymptotic method to fourth order boundary value problems," World Applied Sciences Journal, vol. 9, no. 2, pp. 131-137, 2010.

[8] M. Idrees, S. Islam, S. Haq, and S. Islam, "Application of the optimal homotopy asymptotic method to squeezing flow," Computers \& Mathematics with Applications, vol. 59, no. 12, pp. 38583866, 2010.

[9] M. Idrees, S. Haq, and S. Islam, "Application of optimal homotopy asymptotic method to special sixth order boundary value problems," World Applied Sciences Journal, vol. 9, no. 2, pp. 138$143,2010$.

[10] S. Iqbal, M. Idrees, A. M. Siddiqui, and A. R. Ansari, "Some solutions of the linear and nonlinear Klein-Gordon equations using the optimal homotopy asymptotic method," Applied Mathematics and Computation, vol. 216, no. 10, pp. 2898-2909, 2010.

[11] S. Iqbal and A. Javed, "Application of optimal homotopy asymptotic method for the analytic solution of singular Lane-Emden type equation," Applied Mathematics and Computation, vol. 217, no. 19, pp. 7753-7761, 2011. 


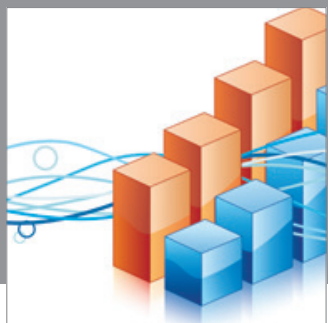

Advances in

Operations Research

mansans

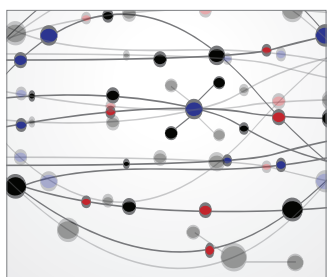

The Scientific World Journal
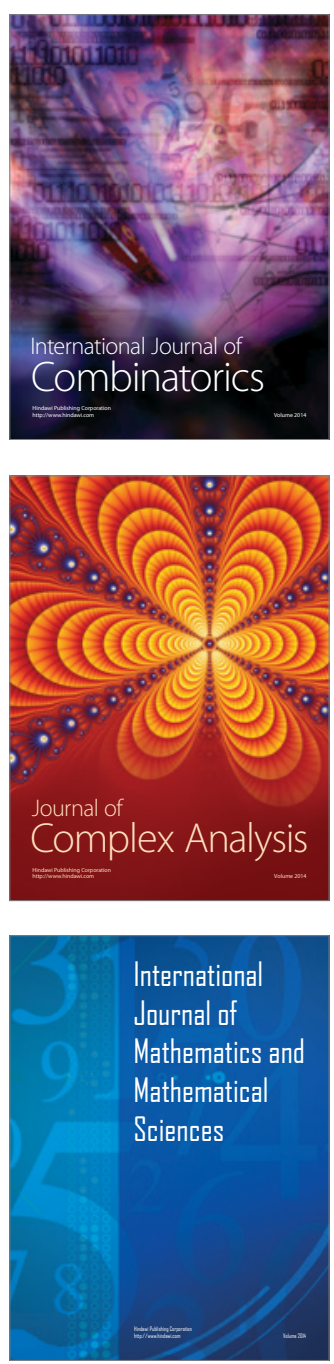
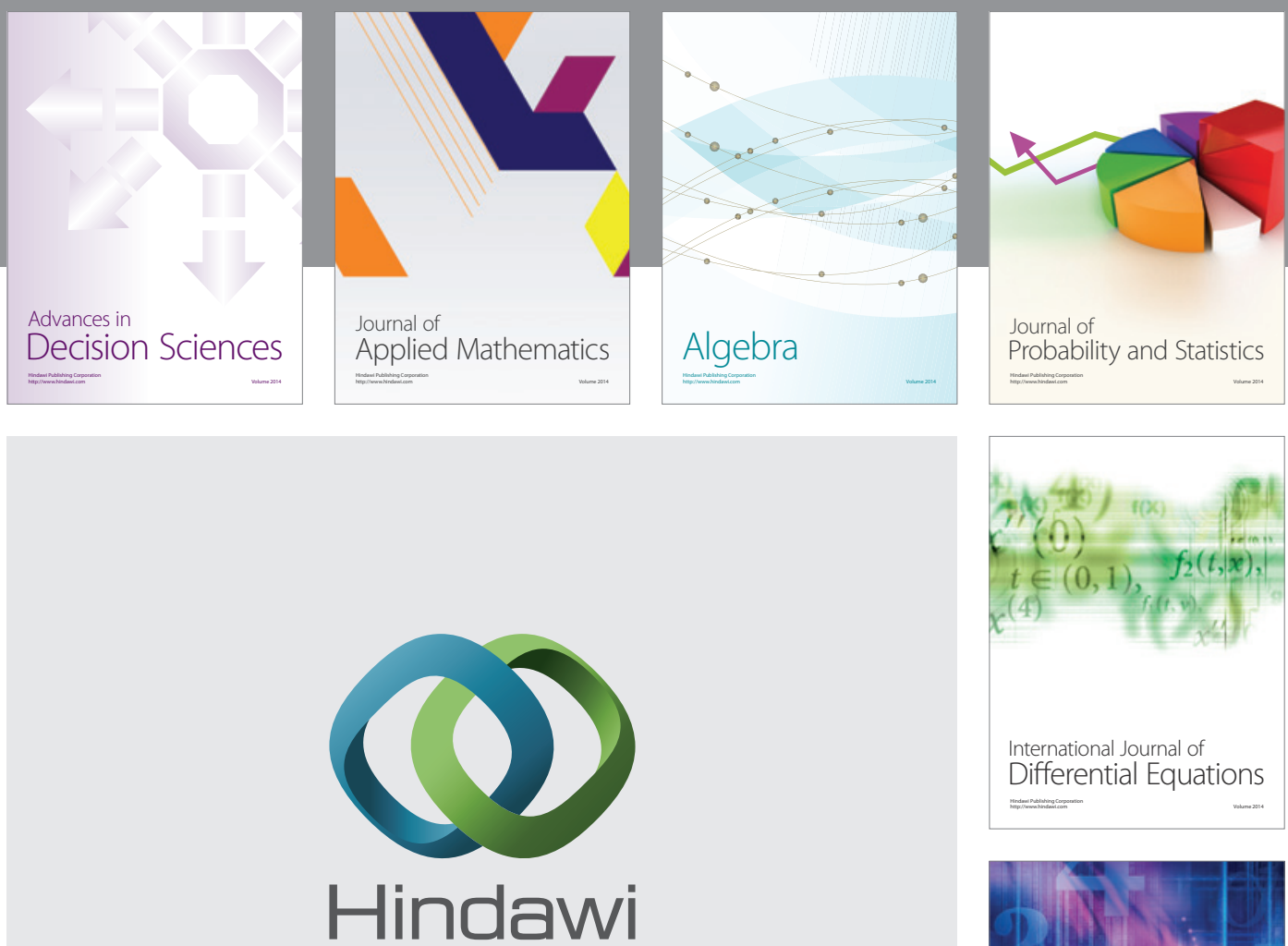

Submit your manuscripts at http://www.hindawi.com
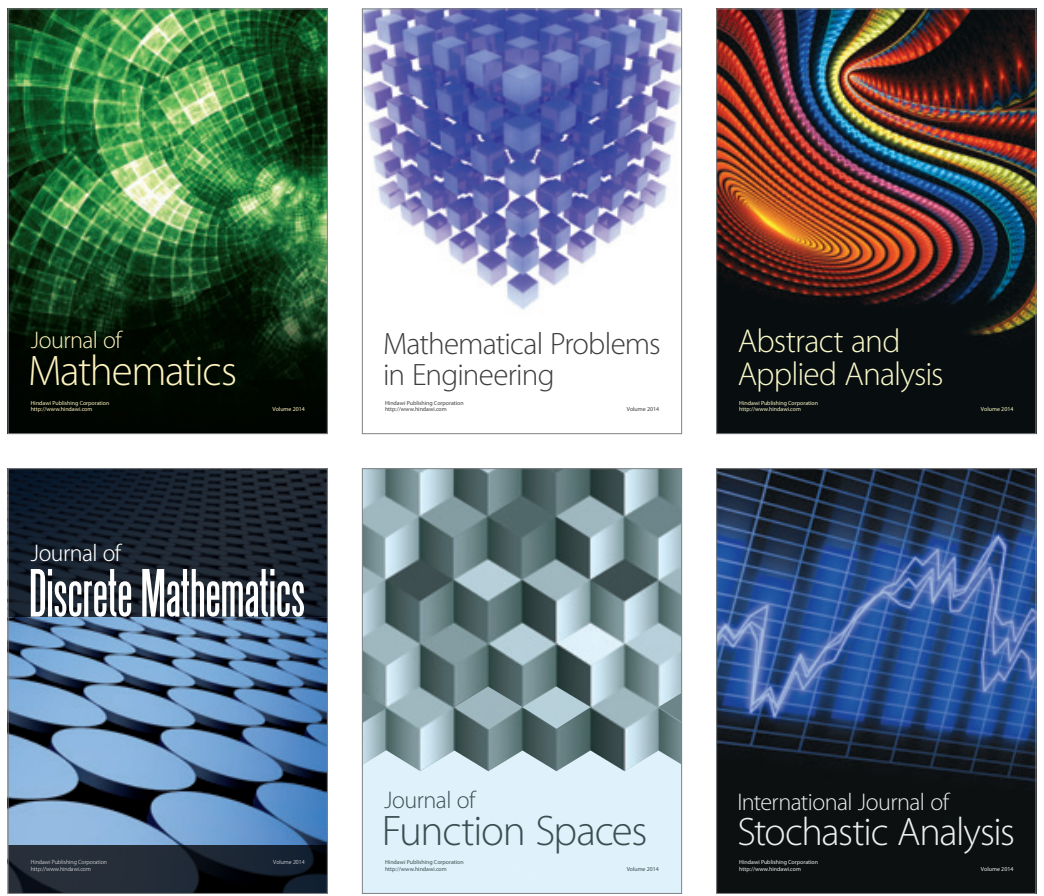

Journal of

Function Spaces

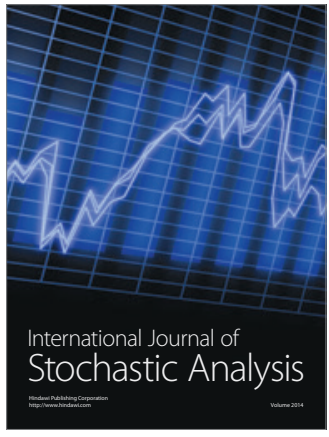

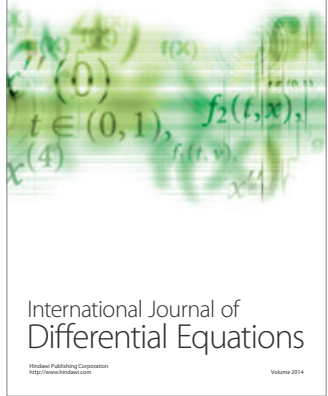
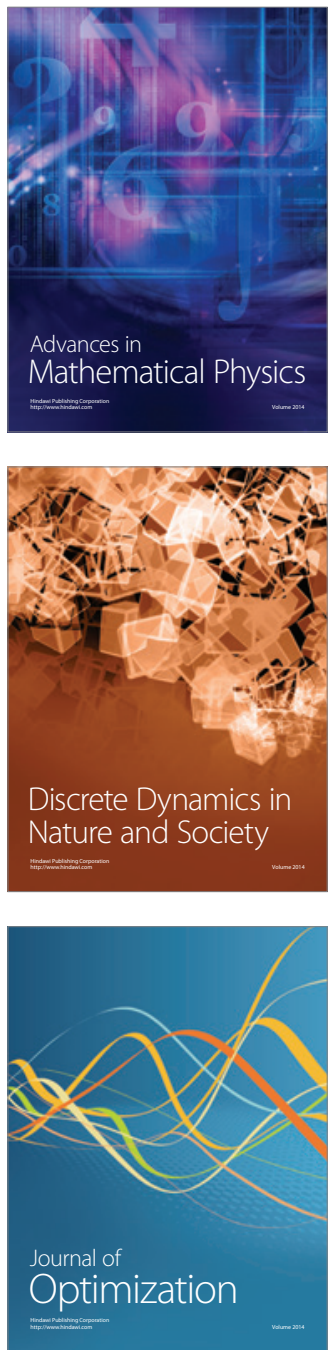